\title{
Ultrasound-guided medial mid-thigh approach to sciatic nerve block with a patient in a supine position
}

\author{
Yoshimune Osaka $\cdot$ Masanori Kashiwagi $\cdot$ \\ Yukio Nagatsuka $\cdot$ Sakurako Miwa
}

Received: 22 July 2010/Accepted: 2 May 2011/Published online: 14 June 2011

(C) Japanese Society of Anesthesiologists 2011

\begin{abstract}
We report the use of a 'medial mid-thigh approach (medial approach), a new approach for performing ultrasound-guided sciatic nerve blockade (SNB) with patients in a supine position. Fifty-four patients undergoing knee surgery under general anesthesia and a combined femoral nerve block (FNB) and SNB were included in the study. After FNB, an ultrasound-guided medial approach was used to perform the SNB. The patient was placed in a supine position, and the hip and knee joints were flexed with the leg rotating externally. A linear ultrasound transducer was positioned perpendicular to the skin at the level of the upper mid-thigh. The sciatic nerve was identified in all patients using ultrasound imaging, and the distance to the nerve was $3.0-5.5 \mathrm{~cm}$. A combined ultrasound- and nerve stimulator-guided SNB was then performed, and $0.375 \%$ ropivacaine was administered. The block was successful in all patients, and the mean duration of the sensory and motor blockade was 11.9 and $8.2 \mathrm{~h}$, respectively. In this study, the medial approach was highly successful and easy to perform. As performing a simultaneous FNB and SNB with patients in a supine position has several potential advantages, future studies should compare this approach with other more proximal approaches for performing SNB.
\end{abstract}

Keywords Sciatic nerve block - Medial mid-thigh approach · Ultrasound guidance - Combined femoral and sciatic nerve block

\footnotetext{
Y. Osaka $(\bowtie) \cdot$ M. Kashiwagi · Y. Nagatsuka · S. Miwa Department of Anesthesiology, Kitasato Institute Hospital, Kitasato University, 5-9-1 Shirokane, Minato-ku, Tokyo 108-8642, Japan e-mail: yosaka@insti.kitasato-u.ac.jp; bluesapphire1226@gmail.com
}

We report a series of 54 patients in whom a 'medial midthigh approach (medial approach)' was successfully used to perform ultrasound-guided sciatic nerve blockade (SNB) while the patient was in a supine position.

Several approaches for SNB have been described [1-9], including an anterior approach [1-3], a posterior approach [35], a popliteal approach [5, 6], a lateral approach [5, 7, 8], and a mid-thigh approach [9]. The anterior approach is useful when the patient cannot be repositioned laterally; however, because a long needle passage is required to reach the sciatic nerve, this technique is technically demanding [1-4]. In addition, a combined femoral nerve blockade (FNB) and SNB is applied under ultrasonographic guidance, changing the linear array (high-frequency) transducer to a low-frequency transducer can be troublesome in some situations.

The purpose of this report was to describe an ultrasoundguided medial approach to the sciatic nerve and to discuss the anatomical details and ultrasonographic features of this approach.

Fifty-four patients undergoing knee surgery were enrolled in this observational trial after the patients had provided written informed consent; institutional ethical board approval was obtained for the study. General anesthesia was induced by administering fentanyl (0.5-1 $\mu \mathrm{g} / \mathrm{kg}$ ) and propofol $(1-2 \mathrm{mg} / \mathrm{kg})$. The anesthesia was maintained using sevoflurane (1-1.5\%) in $40 \%$ oxygen and $60 \%$ nitrous oxide through an inserted laryngeal mask airway, with spontaneous breathing. Following general anesthesia, combined FNB and SNB was performed under ultrasonographic guidance using a hand-held ultrasound device (MicroMaxx Ultrasound System; SonoSite, Bothell, WA) with a high-frequency (6-13 MHz) linear transducer (HFL38/13-6; SonoSite).

The procedures used for the medial approach were as follows. The patient was placed in a supine position with 
the hip and knee flexed and the leg externally rotated by approximately $45^{\circ}$ (Fig. 1a). The ultrasound transducer was positioned perpendicular to the skin at a location approximately $10 \mathrm{~cm}$ distal to the inguinal crease (at the level of the upper middle thigh) and was directed between the adductor magnus muscle (AMM) and the hamstrings (Fig. 1b). A transverse image was obtained of the femur located laterally, with a hyperechoic bone (Fig. 1c) outline and a hypoechoic bone shadow. The transducer was then moved $2-3 \mathrm{~cm}$ posteriorly to visualize the sciatic nerve, which was visible as an oval hyperechoic nodule (Fig. 1d). A short-bevel $(100 \mathrm{~mm})$ 21-gauge insulated nerve block needle (Stimuplex A; B. Braun Melsungen AG, Melsungen, Germany) connected to a nerve stimulator (Contiplex; B. Braun Melsungen AG) was inserted out of plane to the ultrasound transducer. A nerve stimulator with a pulse duration of $0.1 \mathrm{~ms}$ and a stimulating frequency of $2 \mathrm{~Hz}$ was then used to elicit foot movement. The needle was further adjusted as needed to evoke a motor response (eversion, dorsiflexion, inversion, or plantarflexion) when the nerve was stimulated with $0.5-1 \mathrm{~mA}$. We used a nerve stimulator to adjust the tip of the needle and to distinguish the sciatic nerve from the tendons of the semimembranosus and semitendinosus muscles, which appear as hyperechoic structures. Twenty milliliters of $0.375 \%$ ropivacaine was then injected incrementally. Patients received fentanyl $25 \mu \mathrm{g}$ intravenously as needed for adequate pain control.

The depth of the sciatic nerve was measured ultrasonographically and recorded. Time to completion of the blockade from the initiation of the scan to the completion of drug administration was measured. We examined sensory function using a cold test applied to the foot sole and motor function by observing dorsiflexion or plantarflexion of the foot postoperatively. We defined postoperative analgesia in the posterior aspect of the knee, as evaluated by an intensive care unit (ICU) nurse, as a criterion for a successful SNB. The patient data are shown as the mean \pm standard deviation (SD).

The patient characteristics and the block characteristics are presented in Tables 1 and 2, respectively. The sciatic nerve was visible as an oval hyperechoic nodule in all the patients: the nodule was very clearly visible in 39 patients and almost distinctly visible in 15 patients (Fig. 1d). Nerve stimulation was based on a motor response related to the stimulation of the common peroneal nerve branch (dorsiflexion or eversion of the foot) in two patients and the tibial nerve branch (plantarflexion and inversion of the foot and flexion of the toes) in 52 patients. No blood aspiration or sensory or other motor deficits were observed during or after the performance of the SNBs.
Fig. 1 a Patient position, b ultrasound transducer position, $\mathbf{c}, \mathbf{d}$ ultrasound images of the short axis (transverse view) of the sciatic nerve. $S N$ Sciatic nerve, $F$ femur, AMM adductor magnus muscle, $S T M$ semitendinosus muscle, SMM semimembranosus muscle
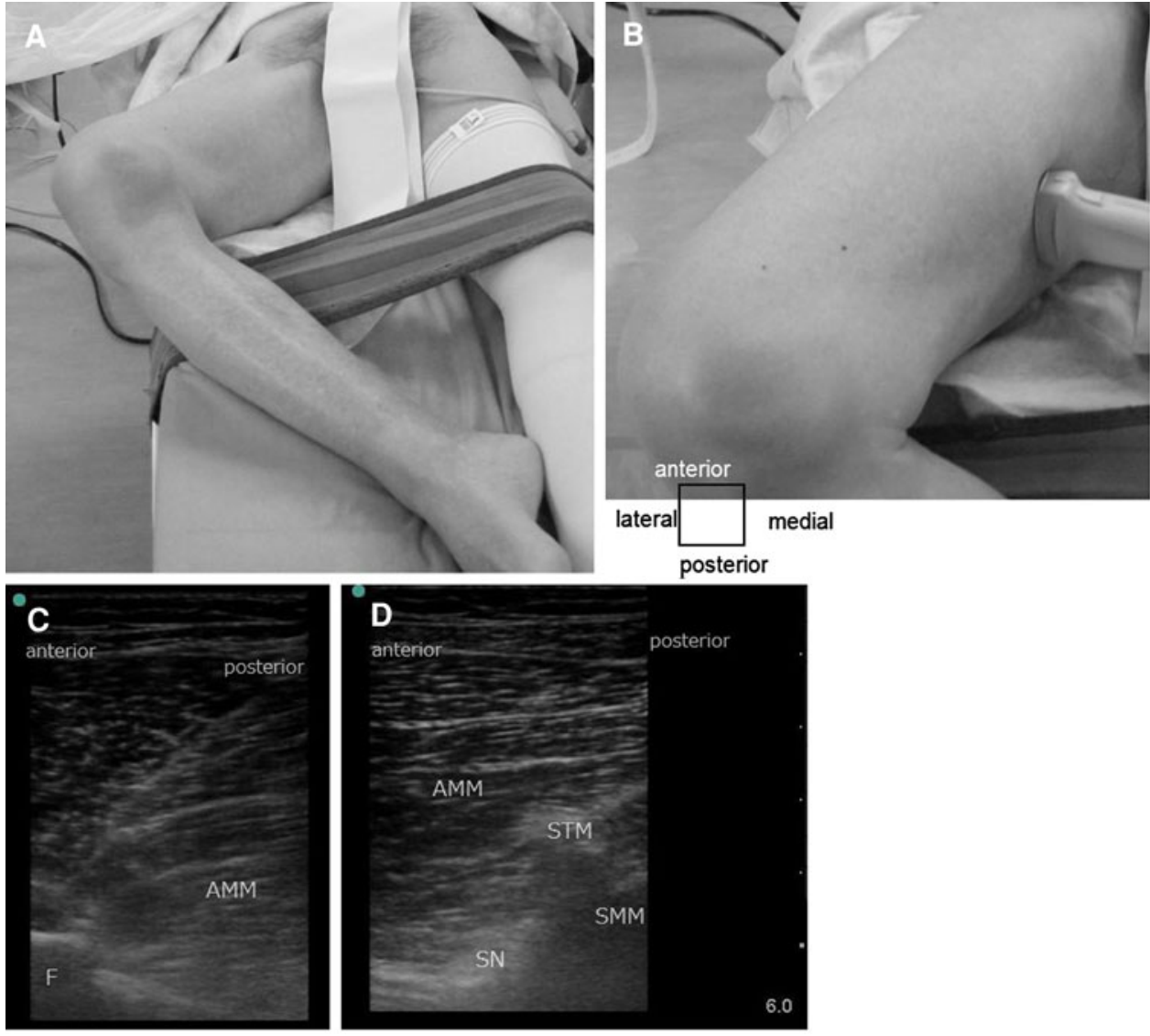
SNB using a medial approach was successful in all the cases. These results show that the SNB can be performed easily and reliably using a linear transducer at the level of the upper middle thigh while the patient is in a supine position.

Several approaches for the SNB have been described [111]. The conventional anterior approach can be performed while the patient is in a supine position [1-5], but it is difficult to perform under ultrasonographic guidance using

Table 1 Patient characteristics

\begin{tabular}{ll}
\hline $\begin{array}{l}\text { Patient } \\
\text { characteristics }\end{array}$ & $\begin{array}{l}\text { Value } \\
(\text { mean } \pm \text { SD) }\end{array}$ \\
\hline Age (year) & $73 \pm 7$ \\
Sex (M/F) & $7 / 47$ \\
Height (cm) & $152 \pm 7$ \\
Weight (kg) & $60.2 \pm 9.8$ \\
BMI & $26.0 \pm 3.3$ \\
\hline
\end{tabular}

$B M I$ body mass index, $F$ female, $M$ male, $S D$ standard deviation

Table 2 Block characteristics and analgesic requirements

\begin{tabular}{lcc}
\hline $\begin{array}{l}\text { Block characteristics } \\
\text { and analgesic requirements }\end{array}$ & $\begin{array}{l}\text { Values } \\
(\text { mean } \pm \text { SD) }\end{array}$ & Range \\
\hline Depth of sciatic nerve $(\mathrm{cm})$ & $4.2 \pm 0.6$ & $3.0-5.5$ \\
Depth of needle insertion $(\mathrm{cm})$ & $5.3 \pm 0.8$ & $4.0-7.0$ \\
Time to completion of blockade (min) & $10.4 \pm 4.5$ & $1-25$ \\
Intraoperative consumption of fentanyl & $151 \pm 50$ & $75-225$ \\
$\quad(\mu \mathrm{g})$ & $11.9 \pm 3.1$ & $6-23$ \\
$\begin{array}{l}\text { Duration of sensory block (h) } \\
\text { Duration of motor block (h) }\end{array}$ & $8.2 \pm 2.3$ & $4-14$ \\
Analgesics requirements $(n)$ & $1.7 \pm 1.0$ & $0-5$ \\
\hline
\end{tabular}

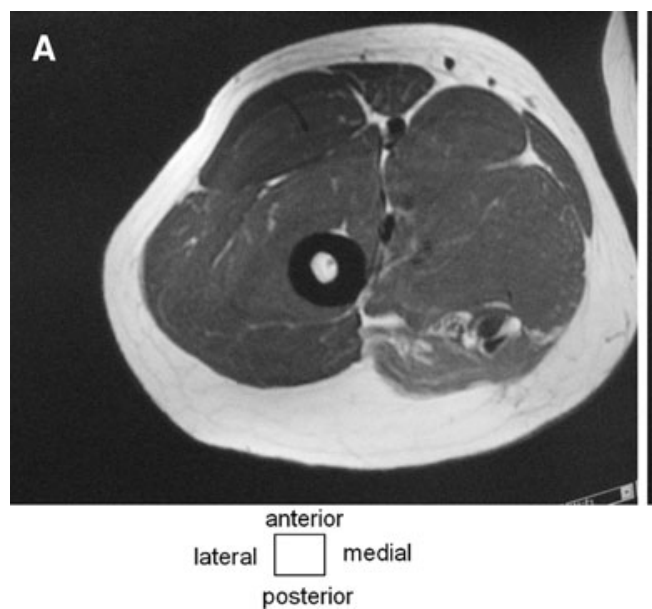

Fig. 2 a Transverse-view of computed tomographic image of the femur at the level of the upper middle thigh. b The trapezoid shows the ultrasonographically imaged area. The sciatic nerve is located superficially in both the medial approach and posterior approach. $S N$ a linear array transducer $[10,11]$. The lateral approach is also difficult to perform using a linear array transducer [7, 8]. The posterior approach [3-5] as well as the mid-thigh approach [9] require that the patient change into a lateral or prone position. By contrast, our approach can be performed with the patient in a supine position.

We were able to obtain a clear image of the sciatic nerve in all patients. Our technique uses two well-defined ultrasonographic landmarks, namely, the AMM on the skin and the femur. The AMM, which is attached to the femur, covers the sciatic nerve [12]. The femur was recognized at a depth of $4-6 \mathrm{~cm}$. The sciatic nerve was recognized in the fat tissue below the AMM at $1.5-2 \mathrm{~cm}$ posterior to the femur at the level of the upper middle thigh, appearing as an oval hyperechoic nodule approximately $1.5-2 \mathrm{~cm}$ in width (Fig. 2). The medial approach has three limitations: (1) the leg must be flexed and rotated; (2) the approach does not enable the blockage of the posterior femoral cutaneous nerve as well as the anterior approach [3, 4]; (3) the visibility of the sciatic nerve is reduced in obese patients.

The use of the same transducer in the same position can be advantageous in terms of cost reduction, total elapsed time of the blocks, degree of discomfort provided by eliminating the need to change the patient's position, and the ease with which the procedure can be performed under general anesthesia.

In conclusion, we propose that the medial mid-thigh approach to SNB is easy and reliable. As performing a simultaneous FNB and SNB while the patient is in a supine position has several potential advantages, future studies should compare this approach with other proximal approaches used for performing SNB.

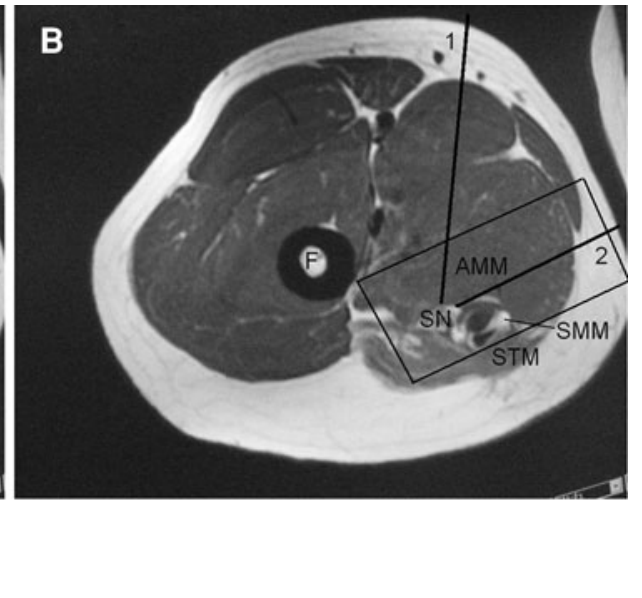

sciatic nerve, $F$ femur, $A M M$ adductor magnus muscle, STM semitendinosus muscle, SMM semimembranosus muscle. Line 1 Needle passage during the anterior approach, Line 2 needle passage during the medial approach 


\section{References}

1. Beck GP. Anterior approach to sciatic nerve block. Anesthesiology. 1963;24:222-4.

2. Chelly JE, Delaunay L. A new anterior approach to the sciatic nerve block. Anesthesiology. 1999;91:1655-60.

3. Ota J, Sakura S, Hara K, Saito Y. Ultrasound-guided anterior approach to sciatic nerve block: a comparison with the posterior approach. Anesth Analg. 2009;108:660-5.

4. Enneking FK, Chan V, Greger J, Hadzić A, Lang SA, Horlocker TT. Lower-extremity peripheral nerve blockade: essentials of our current understanding. Reg Anesth Pain Med. 2005;30:4-35.

5. Brown DL. Sciatic block. In: Brown DL (ed) Atlas of regional anesthesia, 3rd edn. Philadelphia: Elsevier Saunders; 2006. pp. 103-38.

6. Sinha A, Chan VW. Ultrasound imaging for popliteal sciatic nerve block. Reg Anesth Pain Med. 2004;29:130-4.
7. McCartney CJ, Brauner I, Chan VW. Ultrasound guidance for a lateral approach to the sciatic nerve in the popliteal fossa. Anaesthesia. 2004;59:1023-5.

8. Guardini R, Waldron BA, Wallace WA. Sciatic nerve block: a new lateral approach. Acta Anaesthesiol Scand. 1985;29:515-9.

9. Barrington MJ, Lai SL, Briggs CA, Ivanusic JJ, Gledhill SR. Ultrasound-guided midthigh sciatic nerve block-a clinical and anatomical study. Reg Anesth Pain Med. 2008;33:369-76.

10. Vloka JD, Hadzic A, April E, Thys DM. Anterior approach to the sciatic nerve block: the effects of leg rotation. Anesth Analg. 2001;92:460-2.

11. Ericksen ML, Swenson JD, Pace NL. The anatomic relationship of the sciatic nerve to the lesser trochanter: implications for anterior sciatic nerve block. Anesth Analg. 2002;95:1071-4.

12. Moeller TB, Reif E. Pocket atlas of sectional anatomy computed tomography and magnetic resonance imaging, vol 3: spine, extremities, joints. Stuttgart: Thieme Medical Publishers; 2007. pp. 120-35. 